\title{
ON NONTRIVIAL CHARACTERISTIC CLASSES OF CONTACT FOLIATIONS
}

\author{
WEI-LUNG TING ${ }^{1}$
}

\begin{abstract}
In this article we give some nontrivial realizations of secondary characteristic classes of contact foliations.
\end{abstract}

1. Introduction. Given any differentiable pseudogroup $\Gamma$, we can define the $\Gamma$-foliations. In the category of $\Gamma$-foliations one can construct the secondary characteristic classes for $\Gamma$-foliations. The first step in studying characteristic classes is to show that they are nontrivial. Kamber and Tondeur [2] [4] have given many nontrivial realizations of secondary characteristic classes in various geometric contexts. In this article we give some nontrivial characteristic classes for contact foliations.

In $\$ 2$ we recall a construction of the characteristic classes for contact foliations. In $\$ 3$ we compute some of the nontrivial characteristic classes of contact foliations.

2. Contact structures and contact foliations. Let $\left(z, x^{1}, \ldots, x^{n}, y^{1}, \ldots, y^{n}\right)$ be coordinates in $(2 n+1)$-dimensional euclidean space, $R^{2 n+1}$, and let $\gamma$ be the 1 -form on $R^{2 n+1}$ defined by

$$
\gamma=d z+\sum_{i=1}^{n} y^{i} d x^{i}-x^{i} d y^{i}
$$

$\gamma$ is called a contact form on $R^{2 n+1}$, and a diffeomorphism $f: U \rightarrow U^{\prime}$, where $U$ and $U^{\prime}$ are open subsets of $R^{2 n+1}$, is called a contact transformation if and only if $f^{*} \gamma=\tau \gamma$, where $\tau$ is a nonzero real valued function on $U$. The collection $\Gamma$ of all such contact transformations forms a pseudogroup. It is called the contact pseudogroup on $R^{2 n+1}$.

Let $\Gamma$ be the contact pseudogroup on $R^{2 n+1}$. A $\Gamma$-foliation $\mathscr{F}$ of codimension $2 n+1$ on a finite dimensional manifold $M$ is a covering $\left\{U_{i}\right\}$ together with submersion $f_{i}: U_{i} \rightarrow R^{2 n+1}$ that satisfy the condition: for every point $x \in U_{i} \cap U_{j}$ there exists an element $g_{i j} \in \Gamma$ such that $f_{i}=g_{i j} \circ f_{j}$ in some neighborhood of $x$. The collection $\left\{U_{i}, f_{i}\right\}$ is called the atlas of the foliation. Let $\mathscr{F}_{1}$ and $\mathscr{F}_{2}$ be $\Gamma$-foliations on the manifolds $M$ and $N$, respectively. A morphism $\mathscr{F}_{1} \rightarrow \mathscr{F}_{2}$ is a smooth mapping $f: M \rightarrow N$ that satisfies the

Received by the editors January 9, 1978 and, in revised form, September 22, 1978.

AMS (MOS) subject classifications (1970). Primary 57D30.

Key words and phrases. Characteristic classes, Chern-Weil classes, secondary characteristic classes, contact pseudogroups, contact foliations.

${ }^{1}$ The author wishes to express his thanks to the referee for his many helpful suggestions. 
following condition: Let $x \in M$, and let $g_{1}: U \rightarrow R^{2 n+1}$ and $g_{2}: V \rightarrow R^{2 n+1}$ ( $U \subset M, V \subset N$ ) be the mappings of neighborhoods of $x$ and $f(x)$ that belong to the atlases of the foliations $\mathscr{F}_{1}$ and $\mathscr{F}_{2}$, respectively. Then there is a diffeomorphism $\alpha \in \Gamma$ such that $\alpha \circ g_{1}=g_{2} \circ f$ in some neighborhood of $x$.

Let $\Gamma$ be the contact pseudogroup on $R^{2 n+1}$. We define $G^{k}$ to be the set of all $k$-jets of mappings in $\Gamma$ with source and target at 0 . The group $G^{1}$ is called the linear isotropy group of $\Gamma$. Given a $\Gamma$-foliation $\mathscr{F}$ on a manifold $M$, we let $T(\mathscr{F})$ denote the subfibration of $T(M)$ tangent to the leaf of $\mathscr{F}$. We define $P_{\mathscr{F}} \rightarrow M$ to be the principal $G^{1}$-bundle associated with $T(M) / T(\mathscr{F})$.

F. Kamber and P. Tondeur [4] have shown that the principal $G^{1}$-bundle $P_{\mathscr{f}} \rightarrow M$ is a foliated principal bundle. For foliated principal bundles they have the following results.

TheOREM 2.1 [4]. Let $G$ be a Lie group, g the Lie algebra of G. Let $\pi$ : $P \rightarrow M$ be a foliated principal bundle, $H \subset G$ a closed subgroup and $P^{\prime}$ an $H$-reduction of $P$ given by a section $s: M \rightarrow P / H$ of the induced map $\hat{\pi}$ : $P / H \rightarrow M$ :

(i) There is a well-defined multiplicative homomorphism

$$
\Delta_{*}: H \cdot\left(W(\mathrm{~g}, H)_{q}\right) \rightarrow H_{D R}^{\cdot}(M)
$$

where $q$ is the codimension of the foliation on $M, W(\mathrm{~g}, H)_{q}$ the truncated relative Weil algebra. $\Delta_{*}$ is the generalized characteristic homomorphism of $P$.

(ii) $\Delta_{*}$ does not depend on the choice of an adapted connection in $P$. But if $P$ admits a basic connection, then

$$
\Delta_{*}: H^{\cdot}\left(W(\mathrm{~g}, H)_{[q / 2]}\right) \rightarrow H_{D R}(M) .
$$

(iii) $\Delta_{*}$ is functorial under pullback and functorial in $(G, H)$.

(iv) $\Delta_{*}$ is invariant under integrable homotopies.

3. Examples of nontrivial characteristic classes for contact foliations. Throughout this paper we shall use the notations, terminologies, and some results from [2], [4], [5], [7], without always specifying where they come from.

Let us fix an integer $r$ satisfying $0 \leqslant r<[(n-1) / 2]$. Let $P^{n}(C)$ be the $n$-dimensional complex projective space, and let $z_{0}, z_{1}, \ldots, z_{n}$ be the system of its homogeneous coordinates. We define the hermitian matrices $I_{r}$ and $\bar{I}_{r}$ of degree $n-1$ and $n+1$ by

$$
I_{r}=\left(\begin{array}{cc}
-E_{r} & 0 \\
0 & E_{n-r-1}
\end{array}\right) \text { and } \bar{I}_{r}=\left(\begin{array}{ccc}
0 & 0 & \sqrt{-1} \\
0 & I_{r} & 0 \\
-\sqrt{-1} & 0 & 0
\end{array}\right)
$$

where $E_{s}$ is the unit matrix of degree $s$. 
Let $Q_{r}$ be the quadric of $P^{n}(C)$ defined by $\bar{I}_{r}$, that is

$$
\begin{aligned}
Q_{r}=\left\{\left(z_{0}, \ldots, z_{n}\right) \in P^{n}(C) \mid-\sqrt{-1} z_{0} \bar{z}_{n}\right. & -\sum_{i=1}^{r} z_{i} \bar{z}_{i} \\
& \left.+\sum_{i=r+1}^{n-1} z_{i} \bar{z}_{i}+\sqrt{-1} z_{n} \bar{z}_{0}=0\right\} .
\end{aligned}
$$

It is known from [7] that $Q_{r}$ is a connected nondegenerate hypersurface of $P^{n}(C)$ and its index is $r$. We know that $Q_{r}$ has a contact structure of dimension $2 n-1$.

Let $P(n, C)$ be the group of all projective transformations. We consider the subgroup $G(r)$ of $P(n, C)$ which consists of all projective transformations leaving $Q_{r}$ invariant. $G(r)$ acts effectively and transitively on $Q_{r}$ and $G(r)=$ $\mathrm{SU}\left(\bar{I}_{r}\right) / n$, where $n$ is the center of $\mathrm{SU}\left(\bar{I}_{r}\right) . G(r)$ is a connected Lie group. We denote by $G^{\prime}(r)$ the isotropy subgroup of $G(r)$ at $0=(1,0, \ldots, 0) \in Q_{r}$.

The Lie algebra $g(r)$ of $G(r)$ can be identified with $\mathfrak{s u}\left(\bar{I}_{r}\right)$, that is,

$$
\mathrm{g}(r)=\left\{\left.X \in \mathfrak{g l}(n+1, C)\right|^{t} \bar{X} \bar{I}_{r}+\bar{I}_{r} X=0, \text { trace } X=0\right\} ;
$$

$\mathfrak{g}(r)$ is isomorphic to $\mathfrak{s u}(r+1, n-r)$, and so it is simple. Each element $X$ of $\mathrm{g}(r)$ can be written as a matrix of the form

$$
\left(\begin{array}{ccc}
-\bar{u} & -\sqrt{-1}{ }^{t} \bar{w} I_{r} & w_{n} \\
\xi & v & w \\
\xi_{n} & \sqrt{-1}{ }^{t} \bar{\xi} I_{r} & u
\end{array}\right)
$$

where $\xi_{n}, w_{n} \in R, u \in C, \xi, w \in C^{n-1}, v \in u\left(I_{r}\right)$, and $u-\bar{u}+$ trace $v=0$. The Lie algebra $\mathrm{g}(r)$ has a graded Lie algebra structure $\mathrm{g}(r)=\Sigma_{k=-2}^{2} \mathrm{~g}_{k}(r)$, where the $g_{k}(r), k=-2,-1,0,1,2$,are defined as follows: Each element $X$ of $\mathfrak{g}_{-1}(r)$ can be represented as a matrix of the following form

$$
X=\left(\begin{array}{ccc}
0 & 0 & 0 \\
\xi & 0 & 0 \\
0 & \sqrt{-1}{ }^{t} I_{r} & 0
\end{array}\right)
$$

where $\xi \in C^{n-1}$. Each element $X$ of $g_{1}(r)$ can be represented as a matrix of the form

$$
X=\left(\begin{array}{ccc}
0 & -\sqrt{-1}{ }^{t} \bar{\xi} I_{r} & 0 \\
0 & 0 & \xi \\
0 & 0 & 0
\end{array}\right)
$$

where $\xi \in C^{n-1}$. Each element $X$ of $\mathfrak{g}_{-2}(r)$ can be represented as a matrix of 
the form

$$
X=\left(\begin{array}{lll}
0 & 0 & 0 \\
0 & 0 & 0 \\
a & 0 & 0
\end{array}\right)
$$

where $a \in R$. Each element $X$ of $g_{2}(r)$ can be represented as a matrix of the form

$$
X=\left(\begin{array}{lll}
0 & 0 & a \\
0 & 0 & 0 \\
0 & 0 & 0
\end{array}\right)
$$

where $a \in R$. Each element $X$ of $g_{0}(r)$ can be represented as a matrix of the form

$$
X=\left(\begin{array}{ccc}
-\bar{u} & 0 & 0 \\
0 & v & 0 \\
0 & 0 & u
\end{array}\right)
$$

where $u \in C, v \in \mathfrak{u}\left(I_{r}\right)$, and $u-\bar{u}+$ trace $v=0$.

Let $\sigma$ be the involutive automorphism of $g(r)$ which is defined by $\sigma(X)=$ $-{ }^{t} \bar{X}$ for $X \in g(r)$. Then we have $\sigma G(r) \sigma=G(r)$. We define a subgroup $K(r)$ of $G(r)$ by

$$
K(r)=\{X \in G(r) \mid \sigma X \sigma=X\}
$$

and put $K_{0}(r)=K(r) \cap G^{\prime}(r)$. Note that $K(r)$ is compact and $G(r) / G^{\prime}(r)=$ $K(r) / K_{0}(r)=Q_{r}$. The Lie algebra of $K(r)$ is given by

$$
\mathfrak{f}(r)=\{X \in \mathfrak{g}(r) \mid \sigma X=X\}
$$

and $\mathfrak{f}(r)=\mathfrak{m}(r)+\mathfrak{f}_{0}(r)$ (direct sum) where $\mathfrak{f}_{0}(r)=\mathfrak{f}(r) \cap \mathrm{g}_{0}(r)$ is the Lie algebra of $K_{0}(r)$ and $\mathfrak{m}(r)=(\sigma+1) \mathfrak{p}(r)$ where $\mathfrak{p}(r)=\mathfrak{g}_{-2}(r)+\mathfrak{g}_{-1}(r)$. We give some detailed description of the Lie algebra $\mathrm{f}(r)=\mathfrak{f}_{0}(r)+\mathfrak{m}(r)$. The Lie algebra $\mathfrak{f}(r)$ is isomorphic to $\mathfrak{s u}(r+1, n-r) \cap \mathfrak{u}(n+1)$ and the Lie algebra $\mathfrak{f}_{0}(r)$ is isomorphic to $\mathfrak{u}(r) \times \mathfrak{u}(n-r-1)$. We can represent each element $X$ of $\mathrm{f}(r)$ as a matrix of the following form

$$
X=\left(\begin{array}{ccc}
b \sqrt{-1} & -{ }^{t} \bar{\xi} & -a \\
\xi & v & \sqrt{-1} I_{r} \xi \\
a & \sqrt{-1}{ }^{t} \xi I_{r} & b \sqrt{-1}
\end{array}\right\}
$$

where $a, b \in R, \xi \in C^{n-1}, v \in \mathfrak{u}(r) \times \mathfrak{u}(n-r-1)$, and $2 b \sqrt{-1}+$ trace $v$ $=0$. Each element $X$ of $\mathfrak{f}_{0}(r)$ can be represented as a matrix of the following form

$$
X=\left(\begin{array}{ccc}
b \sqrt{-1} & 0 & 0 \\
0 & v & 0 \\
0 & 0 & b \sqrt{-1}
\end{array}\right)
$$


where $b \in R, v \in \mathfrak{u}(r) \times \mathfrak{u}(n-r-1)$, and $2 b \sqrt{-1}+$ trace $v=0$. Each element $X$ of $\mathrm{m}(r)$ can be represented as a matrix of the following form

$$
X=\left[\begin{array}{ccc}
0 & -{ }^{\prime} \bar{\xi} & -a \\
\xi & 0 & \sqrt{-1} I_{r} \xi \\
a & \sqrt{-1}{ }^{\prime} \bar{\xi} I_{r} & 0
\end{array}\right)
$$

where $a \in R, \xi \in C^{n-1}$.

Consider the Lie group $K(r)$ with the Lie subgroup $K_{0}(r) \subset K(r)$ and corresponding Lie algebra $\mathfrak{f}_{0}(r) \subset \mathfrak{f}(r)$. From [2], we have the following results. On the $K_{0}(r)$-bundle

$$
P=K(r) \times K_{0}(r) \rightarrow K(r)
$$

the $K_{0}(r)$-orbits of the diagonal $K_{0}(r)$-action on $K(r) \times K_{0}(r)$ defined by $(\bar{k}, k) k^{\prime}=\left(\bar{k} k^{\prime}, k^{\prime-1} k\right)$ for $\bar{k} \in K, k, k^{\prime} \in K_{0}$ lift the $K_{0}(r)$-orbits on $K(r)$ (the left cosets) to $K(r) \times K_{0}(r)$. Thus the bundle (3.1) has a canonical foliated bundle structure. Moreover, the normal bundle $Q_{K_{0}(r)}$ of the homogeneous foliation $L_{K_{0}(r)}$ on $K(r)$ given by $K_{0}(r)$ (see [4]) is associated to $P$, and the canonical foliation on $Q_{K_{0}(r)}$ is inherited from the canonical foliation of $P$. Thus from here we see that $K(r)$ has a contact foliation structure of codimension $2 n-1$.

Now we consider the exact sequence

$$
0 \rightarrow \mathfrak{f}_{0}(r) \rightarrow \mathfrak{f}(r) \rightarrow \mathfrak{f}(r) / \mathfrak{f}_{0}(r) \rightarrow 0 .
$$

Since $\mathfrak{f}(r)=\mathfrak{f}_{0}(r)+\mathfrak{m}(r)$, we define a map $\theta$ as the projection: $f(r) \rightarrow \mathfrak{f}_{0}(r)$. It is easy to verify that $\theta$ is a $K_{0}(r)$-equivariant splitting of the exact sequence. From [2] we know that $\theta$ defines a $L_{K_{0}(r)}$-basic connection of the $K_{0}(r)$ principal bundle (3.1).

In order to compute the map $\Delta_{*}$ in Theorem 2.1 , we will need the following facts from [2], [4].

(I) Let $G \subset \bar{G}$ be Lie groups. The canonical $G$-foliation $L_{G}$ of $M=\bar{G}$ has codimension $q=\operatorname{dim} \overline{\mathrm{g}} / \mathrm{g}$. The $G$-bundle $P=\bar{G} \times G \rightarrow M$ is canonically foliated. Assume that $\theta$ is a $G$-equivariant splitting of the exact sequence

$$
0 \rightarrow \stackrel{\theta}{\stackrel{\leftrightarrow}{\rightarrow}} \overline{\mathrm{g}} \rightarrow \overline{\mathrm{g}} / \mathrm{g} \rightarrow 0 .
$$

Then there exist an $L_{G}$-basic and locally $\bar{G}$-invariant adapted connection $w$ on $P$, and $\Delta(w)$ on the cochain level factorized as follows

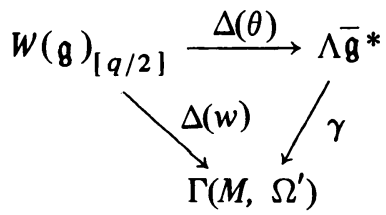


where $\gamma$ is the canonical inclusion and $\Delta(\theta)$ is induced by the $G-D G$ homomorphism

$$
\Delta(\theta): W(\mathfrak{g}) \rightarrow \Lambda \overline{\mathfrak{g}}^{*}
$$

which is completely determined by

$$
\begin{aligned}
\Delta(\theta) \alpha & =\alpha \theta \quad \text { for } \alpha \in \Lambda^{1} \mathrm{~g}^{*}, \\
\Delta(Q) \alpha & =\alpha K(\theta)=d_{\Lambda} \alpha \theta+\frac{1}{2} \alpha[\theta, \theta] \quad \text { for } \tilde{\alpha} \in S^{1} \mathrm{~g}^{*} .
\end{aligned}
$$

(II) Let $(\bar{G}, G)$ be a reductive pair and $\theta: \bar{g} \rightarrow g$ a $G$-equivariant splitting of (3.2). There is a commutative diagram of filtration preserving $G-D G$ homomorphism

$$
\begin{array}{ccc}
W(\mathrm{~g})_{[q / 2]} & \stackrel{\Delta(\theta)}{\rightarrow} & \Lambda \overline{\mathrm{g}}^{*} \\
\varphi \uparrow \simeq & & \varphi \uparrow \simeq \\
\Lambda P_{\mathfrak{g}} \otimes I(G)_{[q / 2]} & \rightarrow & \Lambda P_{\mathfrak{g}} \otimes\left(\Lambda \mathfrak{m}^{*}\right)^{G}
\end{array}
$$

where $\mathfrak{m}=\operatorname{ker} \theta, h(\theta)=\Delta(\theta)_{G}: I(G)_{[q / 2]} \rightarrow\left(\Lambda \mathfrak{m}^{*}\right)^{G}$ is induced by the (ordinary) characteristic homomorphism of $(\bar{G}, G)$ with values in the invariant forms $\left(\Lambda \mathfrak{m}^{*}\right)^{G}$, and where the vertical maps are cohomology isomorphisms.

Now let $(\bar{G}, G)=\left(K(r), K_{0}(r)\right)$. Since $K(r)$ is compact and connected, it is well known that $\gamma_{*}: H^{\cdot}(\mathfrak{f}(x)) \rightarrow H_{D R}(K(r))$ is an isomorphism. By (I) the crucial map to evaluate is the cohomology map $\Delta(\theta)_{*}$. If we identify $f_{0}(r)$ with $\mathfrak{u}(r) \times \mathfrak{u}(n-r-1)$ by the matrices

$$
\left(\begin{array}{lll}
\lambda & 0 & 0 \\
0 & v & 0 \\
0 & 0 & \lambda
\end{array}\right)
$$

with $v \in \mathfrak{u}(r) \times \mathfrak{u}(n-r-1), \lambda=-\frac{1}{2}$ trace $v$, then

$$
I\left(K_{0}(r)\right) \cong R\left[c_{1}, c_{2}, \ldots, c_{n-1}\right] \text { and } I(K(r)) \cong R\left[\bar{c}_{2}, \ldots, \bar{c}_{n+1}\right]
$$

where the $c_{i}, i=1,2, \ldots, n-1$ (resp. $\bar{c}_{j}, j=2, \ldots, n+1$ ) are Chern polynomials. The restriction map $i^{*}: I(K(r)) \rightarrow I\left(K_{0}(r)\right)$ is given by

$$
\begin{array}{ccc}
I(K(r)) & \stackrel{i^{*}}{\rightarrow} & I\left(K_{0}(r)\right) \\
\Downarrow & & \Downarrow \\
R\left[\bar{c}_{2}, \ldots, \bar{c}_{n+1}\right] & \rightarrow & R\left[c_{1}, \ldots, c_{n-1}\right],
\end{array}
$$

$i^{*} \bar{c}_{i+1}=c_{i+1}-c_{1} c_{i}+\frac{1}{4} c_{1}^{2} c_{i-1}$, for $i=1,2, \ldots, n-2, i^{*} \bar{c}_{n}=\frac{1}{4} c_{1}^{2} c_{n-2}-$ $c_{1} c_{n-1}$ and $i^{*} \bar{c}_{n+1}=\frac{1}{4} c_{1}^{2} c_{n-1}$.

Let $y_{i}=\sigma c_{i}$ be the suspension of $c_{i}, i=1,2, \ldots, n-1$. Consider the element $\bar{c}_{n+1}$, a transgressive cochain $T i^{*} \bar{c}_{n+1}$ of $i^{*} \bar{c}_{n+1}=\frac{1}{4} c_{1}^{2} c_{n-1}$ can be obtained in the following way. The cochain

$$
z_{n+1}^{\prime}=y_{n-1} \otimes \frac{1}{4} c_{1}^{2} \in \Lambda\left(y_{1}, \ldots, y_{n}\right) \otimes R\left[c_{1}, \ldots, c_{n-1}\right]
$$


clearly satisfies

$$
d z_{n+1}^{\prime}=1 \otimes \frac{1}{4} c_{1}^{2} c_{n-1}=1 \otimes i^{*} \bar{c}_{n+1}
$$

and hence $\varphi\left(z_{n+1}^{\prime}\right) \in W\left(\mathrm{f}_{0}(r)\right)$ is a transgressive cochain $T i^{*} \bar{c}_{n+1}$ for $i^{*} \bar{c}_{n+1}$. But then by Proposition 5.22 of [2] and by naturality of $\varphi$ we obtain the suspension of $\bar{c}_{n+1}$

$$
\begin{aligned}
\bar{\sigma} \bar{c}_{n+1} & =\left[\Delta(\theta) T i^{*} \bar{c}_{n+1}-\left(\lambda^{1} \bar{c}_{n+1}\right)^{2 n+1,0}\right]=\left[\Delta(\theta) T i^{*} \bar{c}_{n+1}\right] \\
& =\left[\Delta(\theta) \varphi\left(z_{n+1}^{\prime}\right)\right]=\left[\varphi\left(z_{n+1}\right)\right] \in H^{\cdot}(\overline{\mathrm{g}})
\end{aligned}
$$

where

$$
\bar{\pi}_{G}\left(\lambda^{1} \bar{c}_{n+1}\right)=\left(\lambda^{1} \bar{c}_{n+1}\right)^{2 n+1,0} \in\left(\Lambda^{2 n+1} \mathfrak{m}(r)\right)^{G}=0
$$

is the component of bidegree $(2 n+1,0)$ of

$$
\lambda^{1} \bar{c}_{n+1} \in W(\overline{\mathrm{g}}, G)=\left(\Lambda \mathrm{m}(r)^{*} \otimes S \overline{\mathrm{g}}^{*}\right)^{G},
$$

and

$$
z_{n+1}=y_{n-1} \otimes \frac{1}{4} h\left(c_{1}^{2}\right) \in \Lambda\left(y_{1}, \ldots, y_{n-1}\right) \otimes\left(\Lambda \mathfrak{m}(r)^{*}\right)^{G} .
$$

Since $\left(\Lambda^{2 n+2} \mathrm{~m}(r)^{*}\right)^{G}$, the cochain $z_{n+1}$ is a cocycle representing the suspension $\bar{y}_{n+1}$ of $\bar{c}_{n+1}$.

The cochain $z_{n+1}^{\prime}=y_{n-1} \otimes \frac{1}{4} c_{1}^{2}$ is a cocycle in $\Lambda\left(y_{1}, \ldots, y_{n-1}\right) \otimes$ $R\left[c_{1}, \ldots, c_{n-1}\right]_{n-1}$. From (3.3) the cocyle $z_{n+1}^{\prime}$ maps under $\Delta(\theta) \sim$ id $\otimes h$ into the cocycle $z_{n+1}$ representing the nontrivial cohomology class

$$
\bar{y}_{n+1} \in H^{\cdot}(\mathfrak{f}(r)) \cong \Lambda\left(\bar{y}_{2}, \ldots, \bar{y}_{n+1}\right) .
$$

Hence we have the following theorem.

THEOREM 3.1. Let $\left(K(r), K_{0}(r)\right)$ be the reductive pair which is defined as above. Then

$$
\bar{y}_{n+1} \in \operatorname{Im} \Delta(\theta)_{*} \subset H^{\cdot}(\mathfrak{f}(r)) \cong \Lambda\left(\bar{y}_{2}, \ldots, \bar{y}_{n+1}\right),
$$

and $\gamma_{*}\left(\bar{y}_{n+1}\right) \in \operatorname{Im} \Delta_{*}$ is a nonzero characteristic class of the contact foliation of $K(r)$ which is induced by the pair $\left(K(r), K_{0}(r)\right)$.

\section{REFERENCES}

1. I. N. Bernshtein and B. I. Rozenfeld, Homogeneous spaces of infinite-dimensional Lie algebra and characteristic classes of foliations, Russian Math. Surveys 28 (4) (1973), 107-142.

2. F. Kamber and P. Tondeur, Non-trivial characteristic invariants of homogeneous foliated bundles, Ann. Sci. Ecole Norm. Sup. 8 (1975), 433-486.

3. $\ldots$ On the linear independence of certain cohomology classes of $B \Gamma$, Studies in Algebraic Topology, Advances in Math. Suppl. Studies 5 (1979), 213-263.

4. Foliated bundles and characteristic classes, Springer Lecture Notes in Math, vol. 493, Springer, New York, 1975. 
5. N. Tanaka, On non-degenerate real hypersurfaces, graded Lie algebra and Cartan connections, Japan J. Math. 2 (1976), 131-190.

6. C. B. Thomas, A classifying space for the contact pseudogroup, preprint.

7. K. Yamaguchi, Non-degenerate real hypersurfaces in complex manifolds admitting large groups of pseudo-conformal trainsformations, Nagoya Math. J. 62 (1976), 55-96.

Department of Mathematics, State Universtty of New York, College of ArTs and Science, Plattsburgh, New York 12901 